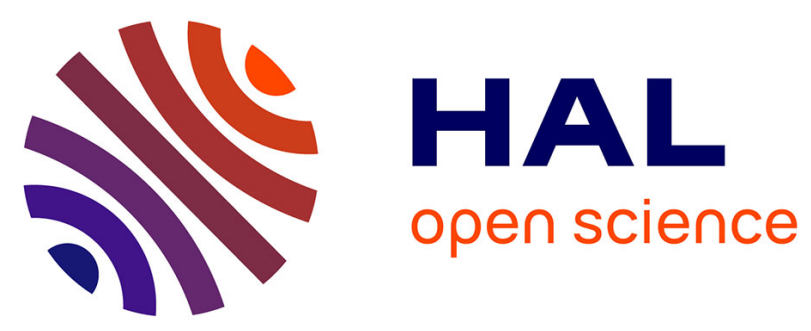

\title{
Generalized self-consistent scheme for the effective behavior of viscoelastic heterogeneous media: A simple approximate solution.
}

H. Hoang-Duc, Guy Bonnet, F. Meftah

\section{- To cite this version:}

H. Hoang-Duc, Guy Bonnet, F. Meftah. Generalized self-consistent scheme for the effective behavior of viscoelastic heterogeneous media: A simple approximate solution.. European Journal of Mechanics - A/Solids, 2013, 29 (1), pp.35-49. hal-00824663

\section{HAL Id: hal-00824663 \\ https://hal.science/hal-00824663}

Submitted on 28 Jan 2016

HAL is a multi-disciplinary open access archive for the deposit and dissemination of scientific research documents, whether they are published or not. The documents may come from teaching and research institutions in France or abroad, or from public or private research centers.
L'archive ouverte pluridisciplinaire HAL, est destinée au dépôt et à la diffusion de documents scientifiques de niveau recherche, publiés ou non, émanant des établissements d'enseignement et de recherche français ou étrangers, des laboratoires publics ou privés. 


\title{
Generalized self-consistent scheme for the effective behavior of viscoelastic heterogeneous media: A simple approximate solution
}

\author{
Hieu Hoang-Duc ${ }^{\mathrm{a}, *}$, Guy Bonnet ${ }^{\mathrm{a}}$, Fekri Meftah ${ }^{\mathrm{b}}$ \\ a Université Paris-Est, Laboratoire Modélisation et Simulation Multi-Echelle, MSME UMR 8208 CNRS, 5 bd Descartes, 77454 Marne-la-Vallée Cedex 2, France \\ ${ }^{\mathrm{b}}$ Laboratoire MMGC, Université Cergy-Pontoise, 5 Mail Gay Lussac, 95031 Cergy-Pontoise Cedex, France
}

\section{A B S T R A C T}

This paper gives a simple approximate solution for obtaining the effective behavior of linear viscoelastic heterogeneous media for the case of elastic inclusions immersed within a viscoelastic matrix. The solution in the Laplace-Carson space is obtained by the Generalized self-consistent model and the simplification is in an explicit expression of the inverse Laplace transform. It is shown that the solution in Laplace-Carson space can be approximated by a convenient rational fraction which is given explicitly as a function of viscoelastic parameters. This provides an easy way to perform the inverse Laplace transform. Examples of typical composites, including possibly void and rigid inclusions, are provided and show that the procedure provides reasonably accurate results. In addition, a complete rheological representation can be provided in some cases for describing the behavior of the effective medium.

Keywords:

Viscoelasticity

Micromechanics

Laplace transform

Generalized self-consistent scheme

Effective properties

\section{Introduction}

Modeling the effective viscoelastic behavior of composite materials by using homogenization methods has been effected by different ways. A classical way is to combine the use of the correspondence principle (Mandel, 1966) with homogenization solutions for elastic composite media. Then, the behavior of the viscoelastic composite is obtained by effecting the inverse Laplace transform (Lee, 1955). Such results were obtained for the Mori and Tanaka (1973) scheme by Wang and Weng (1992), Brinson and Lin (1998), Le et al. (2007) etc. However, it is well known that the Mori-Tanaka's model cannot be used for high concentration of inclusions. In this case, the Generalized self-consistent model is generally preferred. The Generalized self-consistent scheme (Herve and Zaoui, 1993; Christensen and Lo, 1979) was used by Rougier et al. (1994), Beurthey and Zaoui (2000), Masson et al. (2012). The main problem in this last case is that performing the inverse Laplace transform becomes arduous because the expressions of the effective properties in the Laplace space are not rational fractions as functions of the Laplace variable. In this case, more sophisticated means must be used to perform the inverse transform. For example, Padé's approximation was used by Selivanov and Chernoivan

\footnotetext{
Corresponding author. Tel.: +33160957796.

E-mail address: hieuhd80@yahoo.com (H. Hoang-Duc).
}

(2007), the collocation method by Schapery (1962) followed by others (Rekik and Brenner, 2011), while a method for obtaining the relaxation spectra by using Bromwich integration contour was provided by Rougier et al. (1994). The inverse Laplace transform can be also performed by using a semi-analytical computation (Donolato, 2002).

In this paper, a simple, straightforward method is presented in Section 3 for obtaining a suitable approximation of the effective behavior in the Laplace-Carson space, allowing to perform easily the inverse Laplace transform. The classical solution of the Generalized self-consistent scheme is recalled in Section 2. The approximate solution in Laplace space is presented in Section 3. Section 4 is devoted to the presentation of different results in the case of composites containing elastic inclusions within a viscoelastic matrix. Results are compared with those obtained by the semianalytical method of Donolato (2002) and with those obtained from Bromwich integration contour (Rougier et al., 1994).

\section{Generalized self-consistent scheme and its solution in Laplace-Carson space}

2.1. Constitutive equations for an isotropic viscoelastic medium

In the following, a viscoelastic composite material is studied where the phases are non-aging viscoelastic materials or elastic ones. The constitutive stress-strain relation for a non-aging viscoelastic 
material is given classically (Christensen and Freund, 1984; Salençon, 2009) by a Stieljes integral as:

$\boldsymbol{\sigma}(t)=\int_{0}^{t} \mathbb{R}(t-\tau): \frac{\mathrm{d} \varepsilon(\tau)}{\mathrm{d} \tau} \mathrm{d} \tau=\mathbb{R}(: \circledast) \varepsilon$

or reversely:

$\varepsilon(t)=\int_{0}^{t} J(t-\tau): \frac{\mathrm{d} \boldsymbol{\sigma}(\tau)}{\mathrm{d} \tau} \mathrm{d} \tau=\mathbb{}(: \circledast) \boldsymbol{\sigma}$

where $\mathbb{R}, \rrbracket$ are tensorial relaxation and creep functions. The convolution of two functions $f$ and $g$, denoted by " $f \circledast g$ ", is defined by:

$(f \circledast g)(x)=\int^{+\infty} f(x-t) g(t) \mathrm{d} t$

For a viscoelastic isotropic material, tensor $\mathbb{R}$ depends only on two scalar functions $R_{\kappa}(t)$ and $R_{\mu}(t)$ which are relaxation functions for compression and shear. The behavior of the material can be expressed by using the following form:

$\boldsymbol{\sigma}(t)=R_{\kappa}(t) \circledast \operatorname{tr} \varepsilon(t) 1+2 R_{\mu}(t) \circledast \mathbf{e}(t)$

where $\mathbf{e}$ is the deviator of strain tensor.

\subsection{Laplace-Carson transform}

The Laplace-Carson transform $f^{*}(s)$ of a real function $f(t), t \geq 0$ is obtained from its Laplace transform $\tilde{f}(s)$ by:

$f^{*}(s)=s \tilde{f}(s)=s \int_{0}^{\infty} e^{-s t} f(t) \mathrm{d} t$

Effecting the Laplace-Carson transform of the first expression in (4) leads to:

$\boldsymbol{\sigma}^{*}(s)=R_{K}^{*}(s) \operatorname{tr}^{*}(s) 1+2 R_{\mu}^{*}(s) \mathbf{e}^{*}(s)$

which provides also:

$\varepsilon^{*}(s)=\frac{1}{9 R_{K}^{*}(s)} \operatorname{tr} \sigma^{*}(s) 1+\frac{1}{2 R_{\mu}^{*}(s)} s^{*}(s)$

where $s$ is the variable in Laplace-Carson space.

These expressions show that for any fixed value of $s$, the stressstrain relation in Laplace-Carson space is formally equivalent to the elasticity constitutive equation of an isotropic linearly elastic material.

\subsection{The inverse Laplace transform}

The most sophisticated way to perform the inverse Laplace transform of $\tilde{f}(s)$ is to use the Bromwich integration contour (when possible). This method can be avoided in the case where $\tilde{f}(s)$ is a rational function. In this case, the expression of the inverse Laplace transform is recalled in Appendix A. An approximate semianalytical solution which can be used for any expression of $\tilde{f}(s)$ is presented by Donolato (2002). For a real function $f(t), t \geq 0$ and its Laplace-Carson transform $f^{*}(s)$, the approximate expression of the inverse transform is given by: $f_{n}(t)=\frac{1}{n !} \frac{d^{n}}{d u^{n}}\left[u^{n} g(u)\right]$

where $n$ is the order of derivation, $u=t / n+1$ and function $g(u)$ is obtained by the change of variable $s=1 / u$ into function $f^{*}(s)$.

The main problem in the use of this explicit expression is that the solution is obtained at convergence for large values of $n$, while the complexity of derivations increases strongly when the order of derivation is higher, which prevents practically the use of the method for too high orders. This method is called in the following "Differential method".

\subsection{The Generalized self-consistent scheme}

The Generalized self-consistent scheme is based on a composite spherical inclusion immersed into an infinite domain $\Omega$. This composite inclusion is multi-layered as shown in Fig. 1 and characterized by the radii of the spheres $r_{i}, i \in[1, n]$ and by the properties of the constituting materials, namely the incompressibility and shear moduli. All media are considered thereafter as isotropic and characterized by the incompressibility and shear moduli $\left(\kappa_{i}, \mu_{i}\right)$.

Obtaining the effective moduli from the Generalized selfconsistent scheme rests on a fundamental result due to Eshelby (1956) which has shown that the difference of elastic energy between the elastic medium $U$ of the medium with inclusion and the energy $U_{0}$ of the medium without inclusion is given by:

$U=U_{0}-\frac{1}{2} \int_{S}\left(\mathbf{t}^{0} \mathbf{u}^{n+1}-\mathbf{t}^{n+1} \mathbf{u}^{0}\right) \mathrm{d} S$

where $S$ is the interface between inclusion and matrix, $\mathbf{t}^{0}$ and $\mathbf{u}^{0}$ are the traction and displacement on the surface without inclusion while $\mathbf{t}^{n+1}$ and $\mathbf{u}^{n+1}$ are the same quantities for the medium with inclusion. Then, the composite inclusion has the same energy as a volume of material having the effective properties if there is no difference between both energies, leading to:

$\int_{S}\left(\mathbf{t}^{0} \mathbf{u}^{n+1}-\mathbf{t}^{n+1} \mathbf{u}^{0}\right) \mathrm{d} S=0$

This result was used by Christensen and Lo (1979), leading to the effective moduli given thereafter. In addition, the limit values of the effective moduli obtained when the inclusion is voided or rigid are given.

2.4.1. Effective modulus in Laplace-Carson space : general case

Applying the correspondence principle, the effective incompressibility modulus $R_{\kappa}^{*(\text { eff })}$ of a n-phase medium is given from

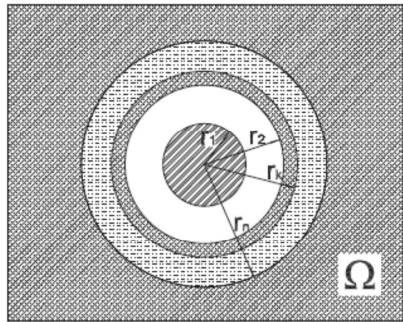

Fig. 1. Schematic representation of the heterogeneous spherical layers in the Generalized self-consistent scheme. 
the properties $R_{K}^{*(i)}$ and $R_{\mu}^{*(i)}(i=1,2 \ldots, n)$ of the constitutive media by:

$R_{\kappa}^{*(\mathrm{eff})}=R_{\kappa}^{*(n)}-\frac{\left(3 R_{\kappa}^{*(n)}+4 R_{\mu}^{*(n)}\right) Q_{21}^{(n-1)}}{3\left(Q_{11}^{(n-1)} r_{n}^{3}+Q_{21}^{(n-1)}\right)}$

where matrix $\mathbf{Q}^{(n-1)}$ is given in Appendix B.1.

The effective shear modulus $R_{\mu}^{*(\text { eff })}$ is the positive root of the quadratic equation:

$A^{*}(s)\left(\frac{R_{\mu}^{*}(\mathrm{eff})}{R_{\mu}^{*(n)}}\right)^{2}+B^{*}(s)\left(\frac{R_{\mu}^{*}(\mathrm{eff})}{R_{\mu}^{*(n)}}\right)+C^{*}(s)=0$

where the "coefficients" $A^{*}(s), B^{*}(s)$ et $C^{*}(s)$ are given as functions of $s$ in Appendix B.2. Under this form, the coefficients of the second order equation are terms in which geometrical parameters and elastic properties are mixed. In the following subsection, another form is provided which allows to write the coefficient of the second order equation as polynomials in the Laplace variable $s$.

2.4.2. A convenient form for the coefficients of the second order equation governing the effective modulus in Laplace-Carson space: case of two phases

It is shown in Appendix B that the previous recurrence relation provides the linear system involving the normalized effective modulus $\bar{\mu}_{\text {eff }}$ :

$\left[\begin{array}{c}\gamma_{0}+3 C_{3}^{\prime} \\ \gamma_{0}-2 C_{3}^{\prime} \\ \bar{\mu}_{\mathrm{eff}}\left(\gamma_{0}-12 C_{3}^{\prime}\right) \\ \bar{\mu}_{\mathrm{eff}}\left(\gamma_{0}+8 C_{3}^{\prime}\right)\end{array}\right]=\mathbf{T}\left[\begin{array}{c}A_{1}^{\prime \prime} \\ B_{1}^{\prime \prime} \\ 0 \\ 0\end{array}\right]$

where all coefficients are unknown constants and where the transfer matrix $\mathbf{T}$ is given below as a function of the ratio $\alpha=r_{2} / r_{1}$ (through matrices $\mathbf{C}_{\mu}$ and $\mathbf{C}_{\kappa}$ given in Appendix B.2) and of the elastic properties of the matrix and of the inclusions.

$\mathbf{T}=\left(\kappa \mathbf{C}_{\kappa}(\alpha)+\mu \mathbf{C}_{\mu}(\alpha)\right)\left(\mu \mathbf{W}_{1}+\mu_{1} \mathbf{W}_{0}\right) \mathbf{L}_{1}^{*}\left(x_{1}\right)$

where $x_{1}$ is a function of the elastic properties of the inclusion and where the pseudo-elastic coefficients $\kappa, \mu$ of the matrix were introduced. The determinant of this homogeneous linear system for unknowns $\gamma_{0}, C_{n+1}^{\prime}, A_{1}^{\prime \prime}, B_{1}^{\prime \prime}$ must be null for having a non-zero solution, which leads to a second order equation where the overall normalized shear modulus $\bar{\mu}_{\text {eff }}$ is obtained by

$A\left(\bar{\mu}_{\text {eff }}\right)^{2}+B \bar{\mu}_{\text {eff }}+C=0$

with:

$\left\{\begin{array}{l}A=4 \Delta_{12} \\ B=\Delta_{23}-2 \Delta_{13}+3 \Delta_{24}-2 \Delta_{14} \\ C=-\Delta_{34}\end{array}\right.$

where $\Delta_{k l}$ is determined from the coefficients of the transfer matrix by:

$\Delta_{k l}=\left|\begin{array}{ll}T_{k 1} & T_{k 2} \\ T_{l 1} & T_{l 2}\end{array}\right|$

Replacing the pseudo-elastic coefficients of the matrix by transforms of the relaxation functions produces:

$$
\begin{aligned}
\mathbf{T}^{*}(s)= & \left(R_{\kappa}^{*(2)}(s) \mathbf{C}_{\kappa}(\alpha)+R_{\mu}^{*(2)}(s) \mathbf{C}_{\mu}(\alpha)\right) \\
& \times\left(\left(R_{\mu}^{*(2)}(s) \mathbf{W}_{1}+R_{\mu}^{*(1)} \mathbf{W}_{0}\right) \mathbf{L}_{1}^{*}\left(x_{1}\right)\right)
\end{aligned}
$$

Under this form, it appears that the coefficients of the secondorder equation are known polynomials of $s$, as soon as the relaxation functions $R_{\kappa}^{*(2)}(s)$ and $R_{\mu}^{*(2)}(s)$ are polynomials or rational fractions.

\section{a) Voided inclusion}

For voided inclusions $\left(k_{1}, \mu_{1}\right) \ll\left(k_{i}, \mu_{i}\right)$ for $[i=2: n]$, matrix $\mathbf{T}^{*}(s)$ in (18) is replaced by $\mathbf{T}_{0}^{*}(s)$ :

$\mathbf{T}_{0}^{*}(s)=R_{\kappa}^{*(2)}(s) \mathbf{C}_{\kappa}(\alpha)+R_{\mu}^{*(2)}(s) \mathbf{C}_{\mu}(\alpha)$

b) Rigid inclusion

For rigid inclusions, $\left(\kappa_{1}, \mu_{1}\right) \gg\left(\kappa_{i}, \mu_{i}\right)$ for $[i=2: n]$. Therefore, the transfer matrix becomes:

$\mathbf{T}_{\infty}^{*}(s)=\left(R_{k}^{*(2)}(s) \mathbf{C}_{k}(\alpha)+R_{\mu}^{*(2)}(s) \mathbf{C}_{\mu}(\alpha)\right) \mathbf{P}$

where $\mathbf{P}$ is a permutation matrix, given by (B.59). The detailed method for providing $\mathbf{T}_{0}^{*}$ et $\mathbf{T}_{\infty}^{*}$ is explained in Appendix B.2.

\section{An approximate solution in real space}

Except in very specific cases, providing closed-form expressions of the inverse Laplace-transform of the values of $R_{*}^{*}(s)$ and $R_{\mu}^{*}(s)$ is not possible. The use of Bromwich integration contour is possible, as shown by Rougier et al. (1994). The construction of the polynomials in the Laplace variable appearing in the expression of the effective modulus would be a significant help for constructing such solutions for various viscoelastic properties. However, its use needs a careful analysis of poles appearing in the expression of the effective modulus and a numerical integration on some parts of the Bromwich contour. So, a more direct approximate solution was preferred in this paper.

Indeed, from another point of view, providing the inverse Laplace transform of a rational fraction of $s$ is possible through algebraical operations. Therefore, a practical way to build the inverse Laplace Transform is to obtain approximations of the previous functions by rational functions. This led for example to use the Padé's approximation, which allows to build an approximation of the Laplace transform by a continued series of rational fractions. This is obviously possible, but the rational fractions contain quickly many terms, which prevents its use at any wished accuracy. The solution which is built thereafter is obtained by using the specific forms of the expressions of $R_{\kappa}^{*}(s)$ and $R_{\mu}^{*}(s)$ provided by the composite sphere model.

The effective incompressibility modulus is given in the LaplaceCarson space by a rational fraction of $s$ and its inverse transform can be obtained from the results of Appendix B.1.

However, the effective shear modulus is given by the solution of a quadratic Equation (12) and has therefore the form:

$R_{\mu}^{*(\mathrm{eff})}(s)=\frac{-B(s) \pm \sqrt{\Delta(s)}}{2 A(s)} R_{\mu}^{n}(s)$

where $A(s), B(s), C(s)$ are polynomials in $s$ given in (B.50) and $\Delta(s)=B(s)^{2}-4 A(s) C(s)$ is also a polynomial in $s$. 
Then, this function will be approached by the approximant $\tilde{R}_{\mu}^{*}(\mathrm{eff})(s)$ which has the form of a rational fraction:

$R_{\mu}^{*(\mathrm{eff})}(s) \approx \tilde{R}_{\mu}^{*}(\mathrm{eff})(s)=\frac{P(s)}{Q(s)}$

where $P(s)$ and $Q(s)$ are both polynomials in $s$. First, it must be noticed that this approximate function must comply with both conditions for extremal values of $s$ :

The initial value condition:

$\lim _{s \rightarrow 0} R_{\mu}^{*(\text { eff })}(s)=\lim _{s \rightarrow 0} \tilde{R}_{\mu}^{*(\text { eff })}(s)$

The ultimate value condition:

$\lim _{s \rightarrow \infty} R_{\mu}^{*(\text { eff })}(s)=\lim _{s \rightarrow \infty} \tilde{R}_{\mu}^{*(\text { eff })}(s)$

The construction of $P(s)$ is achieved by looking for an approximate value of $\Delta(s)$ under a polynomial form. Thus $\Delta(s) \approx T^{2}(s)$, where:

$T(s)=a_{0}+a_{1} s+\ldots+a_{m} s^{m}$

The initial value and ultimate value conditions are satisfied if:

$\lim _{s \rightarrow 0} \frac{T(s)}{2 A(s)} R_{\mu}^{*(n)}(s)=\lim _{s \rightarrow 0} \frac{\sqrt{\Delta(s)}}{2 \tilde{A}(s)} R_{\mu}^{*(n)}(s)$

and:

$\lim _{s \rightarrow \infty} \frac{T(s)}{2 A(s)} R_{\mu}^{*(n)}(s)=\lim _{s \rightarrow \infty} \frac{\sqrt{\Delta(s)}}{2 \tilde{A}(s)} R_{\mu}^{*(n)}(s)$

where :

$\Delta(s)=\Delta_{0}+\Delta_{1} s+\ldots+\Delta_{p} s^{p} \quad(p=2 m)$

The coefficients $\Delta_{i}$ are always positive for any $s$. So, the initial value and ultimate value conditions are satisfied if:

$\left\{a_{0}=\sqrt{\Delta_{0}}\right.$

$a_{m}=\sqrt{\Delta_{p}}$

All the coefficients of $T(s)$ are then obtained as follows: ideally, the square of the polynomial $T(s)$ should approximate $\Delta$. So:

$T(s)^{2}=\sum_{i=0}^{p} b_{i} s^{i} \approx \Delta(s)$

leading to a sequence of $p+1$ equations:

$\Delta_{i}=b_{i}= \begin{cases}\sum_{r=0}^{i} a_{r} a_{i-r} & (i \leq m) \\ \sum_{r=i-m}^{p-m} a_{r} a_{i-r} & (i>m)\end{cases}$

Obviously, it is not possible to comply with these equations in totality, because the system is overdetermined. Taking into account that two of these equations are verified by the initial value and ultimate value conditions, $m-1$ supplementary equations can be used to provide the coefficients of $T(s)$. Different strategies can be adopted, either pseudo-inverse solutions or using only a set of equations having a number equal to the one of unknown terms. The use of pseudo-inverse does not respect the initial and ultimate value conditions and therefore it was not efficient. Different tests

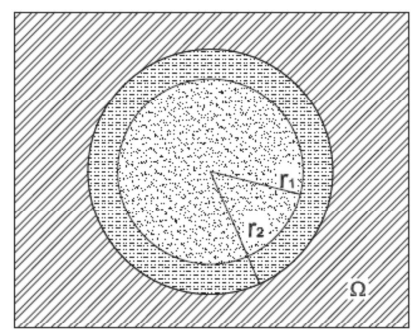

Fig. 2. A two-phase composite inclusion.

were effected and a simple way producing satisfying solutions, when compared with the solution provided by the Bromwich contour, was to use the equations coming from higher terms and lower terms of $\Delta(s)$ in equal quantity if the number of coefficients of $T(s)$ is even. If the number of terms is odd, one more term is kept, chosen as the one producing the solution nearest to the solution of second-order equations between the subsequent higher term and the subsequent lower term.

It means that initial and ultimate conditions are verified upto the highest possible order in the asymptotic expansion. This leads (for a degree $m$ of $T(s)$ being even, which was the case for all examples of this paper) to use the first set of Equation (31) for $i=0 \ldots 1 m_{1}$ (with $m_{1}=m / 2-1$ or $m_{1}=m / 2$ ) and the second set of equations for $i=m_{2} \ldots 2 m$ (with $m_{2}=3 m / 2$ or $m_{2}=3 m / 2+1$ ).

In the following, the approximate solution using the procedure described in this section based on a Rational Fraction (RF) will be called "RF approximate solution".

\section{Application for a composite containing a matrix made of} a Maxwell viscoelastic material

The application is performed in this section in the case of elastic inclusions surrounded by a matrix which is made of a Maxwell viscoelastic material for which the relaxation functions in the Laplace-Carson space are given by Equation (C.10) of Appendix C:

$\left\{\begin{array}{l}R_{\kappa}^{*}=\frac{\kappa \eta_{\kappa} s}{\kappa+\eta_{\kappa} s} \\ R_{\mu}^{*}=\frac{\mu \eta_{\mu} s}{\mu+\eta_{\mu} s}\end{array}\right.$

The Generalized self-consistent scheme is therefore used with the two-phase composite inclusion given in Fig. 2.

The mechanical properties of the components used for this example are given in Table 1.

The results obtained by the proposed method will be compared with the solution of the differential method of Donolato and with the solution of Beurthey and Zaoui obtained from the Bromwich

Table 1

Volume fractions and material properties of the constituents of a 2-phase viscoelastic composite with one constituent of Maxwell type. Phase 1 contains the inclusions while phase 2 contains the matrix.

\begin{tabular}{lcc}
\hline Material phases & Phase 1 & Phase 2 \\
\hline Volume fraction (\%) & 37 & 63 \\
Elastic properties & & \\
$\quad$ Bulk modulus $\kappa$ (GPa) & 100 & 15 \\
$\quad$ Shear modulus $\mu$ (GPa) & 20 & 2 \\
Viscous properties & 0 & 24 \\
$\quad$ Bulk viscosity $\eta_{\kappa}$ (GPa.day) & 0 & 18 \\
$\quad$ Shear viscosity $\eta_{\mu}$ (GPa.day) & &
\end{tabular}


contour integral (Beurthey and Zaoui, 2000; Rougier et al., 1994). The effective shear modulus which is given by (21) is split into two parts:

$R_{\mu}^{* \text { eff }}=f_{1}^{*}(s) \pm f_{2}^{*}(s)$

with:

$f_{1}^{*}(s)=-\frac{B(s)}{2 A(s)} R_{\mu}^{2}(s)$

$f_{2}^{*}(s)=\frac{\sqrt{\Delta(s)}}{2 A(s)} R_{\mu}^{2}(s)$

where the first term $f_{1}^{*}(s)$ is a rational fraction in $s$, so that, $f_{1}(t)$ is given by Appendix A. $\Delta(s), A(s)$ are fourth-order and second-order polynomials, defined by Appendix B.2, which can be rewritten as:

$$
\begin{aligned}
& \Delta(s)=\prod_{i=1}^{8}\left(s+\frac{1}{\tau_{i}}\right) \\
& A(s)=\prod_{j=1}^{4}\left(s+\frac{1}{\phi_{j}}\right)
\end{aligned}
$$

where $-1 / \tau_{i},-1 / \phi_{j}$ are the distinct real roots of $\Delta(s)$ and $A(s)$, respectively, which are numerically determined.

The inverse Laplace transform using the Bromwich integral for $f_{2}^{*}$ is written in Beurthey and Zaoui (2000), Rougier et al. (1994) under the form of the sum of exponential terms and of real integrals on bounded intervals which are functions of $\tau_{i}$ and $\phi_{j}$. The solution using the Bromwich integral is considered as an "exact solution" since the integral on a finite interval can be easily computed upto any wished accuracy.

The quality of the RF approximate solution depends obviously on the ability of RF expression to approximate the solution of the second order equation (exact solution) in Laplace-Carson space. So, Fig. 3 shows the comparison between the shear moduli obtained in the Laplace-Carson space from the exact solution and from the approximate solution while Fig. 4 shows the relative difference between both expressions given by the relation

$\mathrm{Err}=\frac{R_{\mu}^{*(\mathrm{eff})}(s)-\tilde{R}_{\mu}^{*(\mathrm{eff})}(s)}{R_{\mu}^{*}(\mathrm{eff})(s)}$

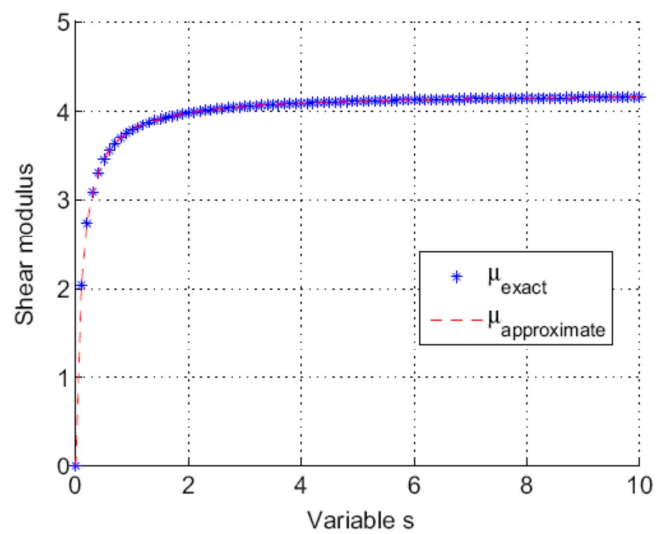

Fig. 3. Comparison between the exact solution and the RF approximate solution in Laplace-Carson's space for a Maxwell viscoelastic matrix containing elastic inclusions.

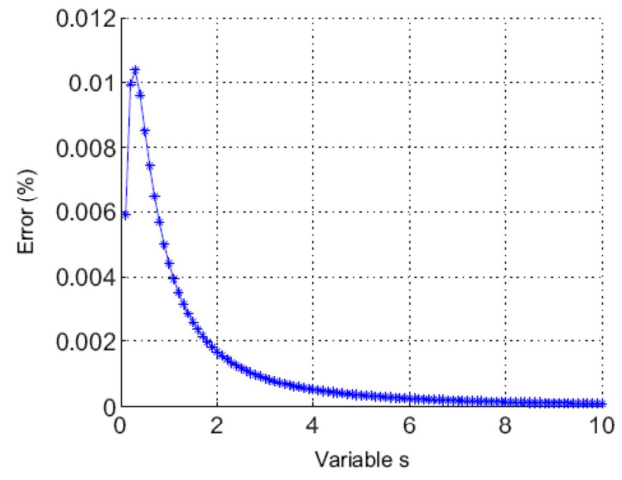

Fig. 4. Error evaluation coming from the RF approximate method in Laplace-Carson's space for a Maxwell viscoelastic matrix containing elastic inclusions.

These figures show that the approximate RF solution allows to recover the solution in the Laplace-Carson space with an error around $1 \cdot 10^{-4}$

This comparison shows that the relaxation function coming from rational fraction approximates correctly the solution in Laplace-Carson space. So, a comparison is now made in the real space after performing the inverse Laplace transform. This inverse Laplace transform has been effected by using the RF approximate solution, the exact solution using the integral on Bromwich contour (Beurthey and Zaoui, 2000; Rougier et al., 1994) and the differential method at different orders.

Fig. 5 shows the relaxation functions coming after Laplace inverse from these different procedures. It can be seen that the RF approximate solution is very close to the exact solution, while a difference with the solution coming from the differential method persists even for an order of derivation $n=5$ in the differential method, which is at the limit of the use of such a method for the considered problem.

It implies that the RF approximate solution is clearly better than the solution coming from the differential method. This result can be confirmed by comparing the Laplace inverse of the RF solution with the one coming from the Laplace inverse computed by using the

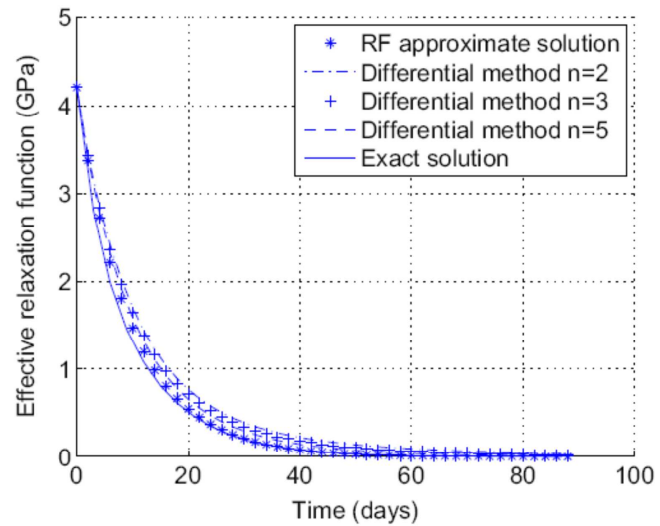

Fig. 5. Relaxation function in real space for a Maxwell viscoelastic matrix containing elastic inclusions. 


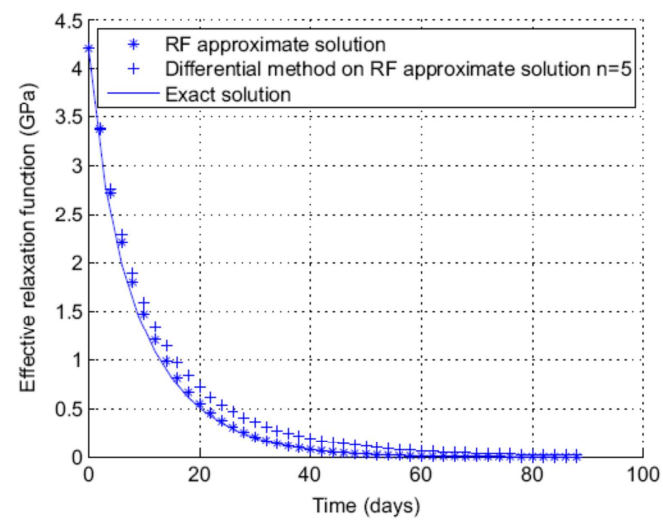

Fig. 6. Relaxation function obtained by the differential method on the RF approximate solution compared to the exact Laplace inverse performed on the RF approximate solution and exact solution for a Maxwell type viscoelastic matrix containing elastic inclusions.

differential method. Indeed, there is no error coming from the Laplace inverse of the RF solution, this one being a rational fraction.

Fig. 6 displays such a comparison. In addition, the exact solution of Fig. 5 has also been reported again. This figure shows clearly again that the error coming from the inversion by the differential method is larger than the difference between the RF approximate solution and the exact solution.

The conclusion coming from these results is that the RF approximate solution produces results which are closer to the exact solution than the differential method upto $n=5$.

In the following, the applications are performed for KelvinVoigt materials where the solution using Bromwich contour are no more available. It is no more possible to assess the correctness of results directly as for Maxwell type materials. However, two comparisons can be still used to qualify the results: the comparison between exact and RF approximate solution in Laplace space and the comparison between exact Laplace inverse of the RF solution with the Laplace inverse of the RF solution coming from the differential method.

\section{Application for a composite containing a matrix made of} a Kelvin-Voigt viscoelastic material

The method described in the previous sections is now used for obtaining the effective properties of a two-phase composite material made of a viscoelastic matrix of the Kelvin-Voigt type containing elastic inclusions. Three cases were studied. The first one is an intermediate case where the contrasts between elastic

Table 2

Volume fractions and material properties of the constituents of a 2-phase composite, one constituent being a Kelvin-Voigt viscoelastic material. Phase 1 corresponds to the inclusions and phase 2 to the matrix.

\begin{tabular}{lll}
\hline Material phases & Phase 1 & Phase 2 \\
\hline Volume fraction (\%) & 40 & 60 \\
Elastic properties & & \\
$\quad$ Bulk modulus $\kappa$ (GPa) & 70 & 0.1 \\
$\quad$ Shear modulus $\mu$ (GPa) & 10 & 0.0375 \\
Viscous properties & & 5 \\
$\quad$ Bulk viscosity $\eta_{\kappa}$ (GPa.day) & 0 & 1 \\
$\quad$ Shear viscosity $\eta_{\mu}$ (GPa.day) & 0 & \\
\hline
\end{tabular}

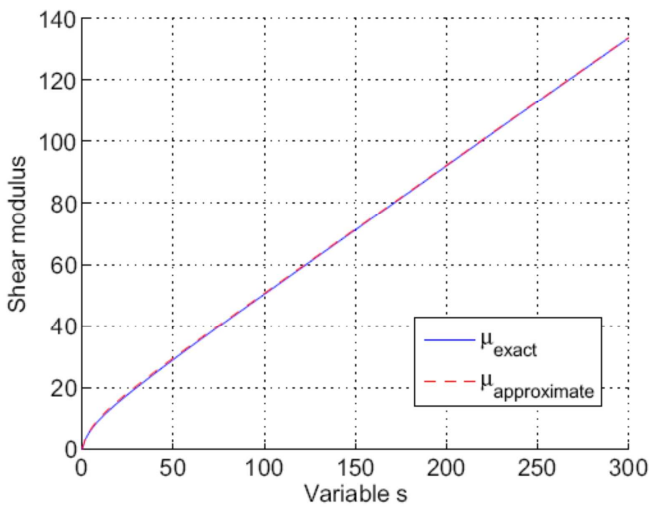

Fig. 7. Comparison between the exact solution and the approximate solution in Laplace-Carson's space for a Kelvin-Voigt viscoelastic matrix containing elastic inclusions.

properties of the matrix and of the inclusion are finite. Next, the case of matrix containing rigid inclusions is presented and finally, the case of voided inclusions is studied.

\subsection{Intermediate case}

In this case, the inclusions are made of a linear elastic material characterized by the incompressibility modulus $\kappa_{1}$ and by the shear modulus $\mu_{1}$. The matrix is made of a Kelvin-Voigt viscoelastic material for which the relaxation functions in the Laplace-Carson space are given by Equation (C.10) of Appendix C:

$\left\{R_{\kappa}^{*}=\kappa+\eta_{\kappa} S\right.$

$\left\{R_{\mu}^{*}=\mu+\eta_{\mu} s\right.$

where $\kappa, \mu$ are the instantaneous incompressibility and shear moduli. $\eta_{\kappa}, \eta_{\mu}$ are the bulk and shear viscosities, $s$ is the LaplaceCarson variable.

All physical parameters used for the application are assembled in Table 2.

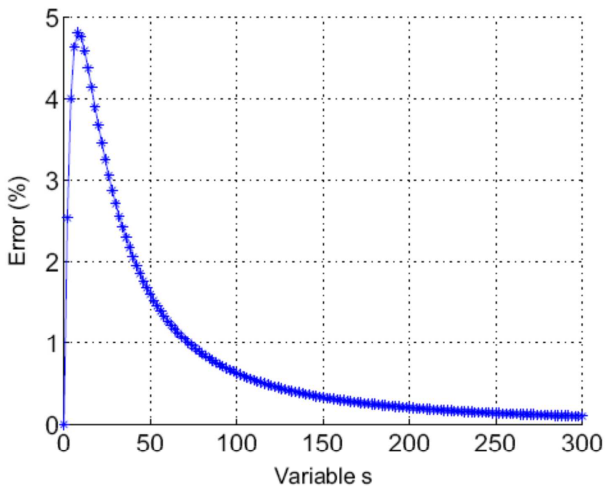

Fig. 8. Evaluation of the relative difference coming from the RF approximate method in Laplace-Carson's space for a Kelvin-Voigt viscoelastic matrix containing elastic inclusion 


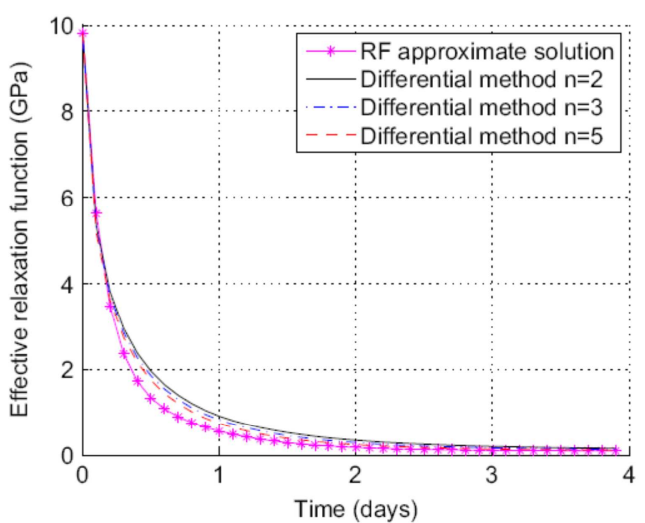

Fig. 9. Relaxation function in real space for the case of a Kelvin-Voigt viscoelastic matrix containing elastic inclusions.

5.1.1. The shear problem

With the previous data, the coefficients $A^{*}(s), B^{*}(s), C^{*}(s)$ within the quadratic polynomial giving the effective shear modulus in Laplace-Carson space are given by the fourth-order polynomials:

$$
\begin{aligned}
A^{*}(s)= & 1.4263 \cdot 10^{-7} s^{4}+1.6118 \cdot 10^{-4} s^{3}+0.0408 s^{2} \\
& +0.4107 s+1 \\
B^{*}(s)= & -3.2260 \cdot 10^{-8} s^{4}-1.1227 \cdot 10^{-4} s^{3}-0.0689 s^{2} \\
& -0.6621 s-1.4502 \\
C^{*}(s)= & -9.5305 \cdot 10^{-9} s^{4}-6.1597 \cdot 10^{-5} s^{3}-0.0583 s^{2} \\
& -0.8568 s-3.1101
\end{aligned}
$$

The comparison between the exact solution and the RF approximate solution in the Laplace-Carson space is given in Fig. 7 which shows a very good agreement. The level of approximation in the Laplace-Carson's space can be estimated by the values of the relative difference, as previously.

The relative difference induced by the use of RF approximate solution in Laplace-Carson space is displayed in Fig. 8 which shows that this difference reaches $5 \%$ at its maximum.

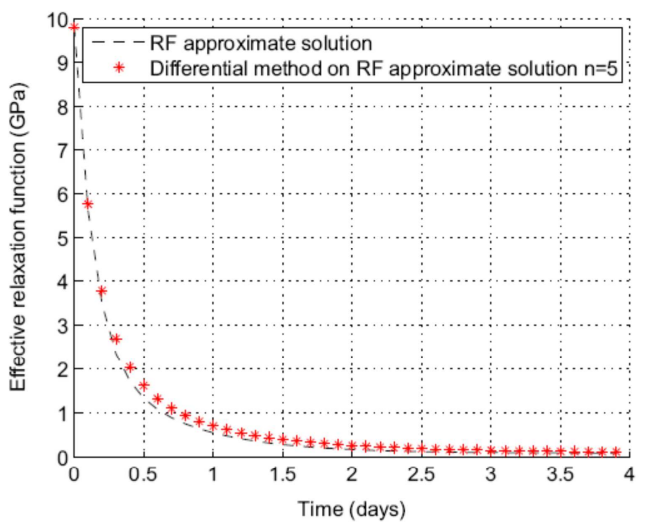

Fig. 10. Relaxation function obtained by the differential method on the RF approximate solution compared to the exact Laplace inverse performed on the RF approximate solution for a Kelvin-Voigt viscoelastic matrix containing elastic inclusions.

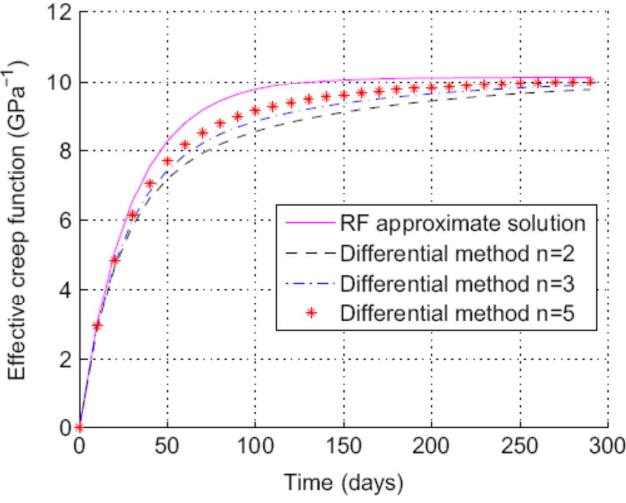

Fig. 11. Creep function in real space of a Kelvin-Voigt viscoelastic matrix containing elastic inclusions.

As previously, the question is now to evaluate the quality of the $\mathrm{RF}$ approximate solution in the real space. To this aim, a comparison is made with results given by the differential method of Donolato (2002) described in Section 2. As explained before, the quality depends of the level of the order of differentiation used in the differential method summarized in (8). Fig. 9 shows that the differential method used at orders 2,3 and 5 gives similar results, both being, as in the previous section, significantly different from those given by the RF approximate method of Section 3.

As in the previous section, the examination of the quality of the solution coming from the differential method is performed by comparing in real space the results coming from th is method when applied to the RF solution with those coming from the direct inverse of the RF solution. Fig. 10 shows this comparison. The results are similar to those obtained for the Maxwell material: the curves are similar and the results coming from the differential method become closer to the RF solution when the degree of differentiation increases. There is however still a notable difference between both solutions for the degree 5, which is the highest degree of differentiation.

The effective creep function which is finally obtained is illustrated in Fig. 11. The results corresponding to the RF approximate solution and those obtained by the differential method are presented, showing again for this new function that a gap appears between both solutions.

From another point of view, due to the structure of its expression, the RF approximate solution presents another advantage: it makes it possible to characterize a rheological model allowing to recover the macroscopic behavior. This rheological model is shown in Fig. 12. Determining the coefficients of the rheological model is indeed given by:

$\left\{\begin{array}{l}\eta_{i}=\frac{1}{R_{i}} \\ E_{i}=\frac{S_{i}}{R_{i}}\end{array}\right.$

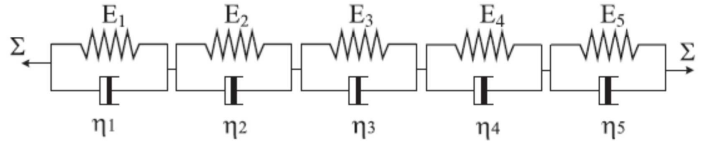

Fig. 12. Rheological model associated with the macroscopic shear behavior 
Table 3

Macroscopics viscoelastics properties of the rheological model associated with the macroscopique shear behavior.

\begin{tabular}{lcc}
\hline Element & $\begin{array}{l}\text { Shear modulus } \\
\text { (GPa) }\end{array}$ & $\begin{array}{l}\text { Viscosity } \\
\text { (GPa.day) }\end{array}$ \\
\hline 1 & 15.78 & 0.53 \\
2 & 16.98 & 5.66 \\
3 & 0.19 & 5.05 \\
4 & 0.26 & 8.09 \\
5 & 1.08 & 47.05 \\
\hline
\end{tabular}

where the coefficients $R_{i}, S_{i}$ are obtained from the decomposition:

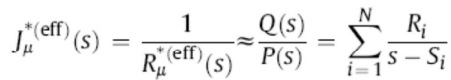

The values which were so obtained are given in Table 3 .

\subsection{Case of the voided inclusion}

In this first extreme case, the model contains a central hole which is surrounded by a matrix with the same behavior of viscoelastic Kelvin-Voigt type as previously. The mechanical properties of each phase are found in Table 4.

As before, the comparison is made between the solution of the second order equation and the RF approximate solution in the Laplace-Carson's space in Fig. 13. Both results correspond to straight lines, indicating that the overall behavior is also close to a model of "Kelvin-Voigt" type, corresponding to the behavior of the matrix. Several authors have indeed found that the type of behavior is identical to the behavior of the matrix, in the case of rigid inclusions or voids. For example, the study by homogenizing the behavior of damaged (containing voids) viscoelastic materials comprising a matrix of type "Burgers" is also of "Burgers" type (Nguyen et al., 2010).

The difference between both curves of Fig. 13 is shown in Fig. 14 On this new example, the difference is less than $1 \cdot 10^{-4}$, which shows a good quality of the RF approximate model. The effective relaxation function is plotted in Fig. 15, where the results obtained by the differential method are also deferred. There remains a gap with the differential method as in the previous result, but the results of the differential method come nearer to the RF solution for increasing values. Finally, Fig. 16 shows the creep functions obtained by the differential method and by the RF approximate method, showing the same trends as previously.

\subsection{Case of rigid inclusions}

In this case, inclusions are assumed rigid and the matrix is again assumed of the Kelvin-Voigt viscoelastic type. All physical properties are given in Table 5. As shown before, the case of isotropic stress loading corresponds to a rational fraction. Then, in the following, only the case of shear loading will be studied.

Table 4

Volume fractions and material properties of the constituents of a 2-phase composite containing voided inclusions.

\begin{tabular}{llc}
\hline Material phases & Phase 1 & Phase 2 \\
\hline Volume fraction (\%) & 67 & 33 \\
Elastic properties & & 100 \\
$\quad$ Bulk modulus $\kappa$ (GPa) & 0 & 20 \\
Shear modulus $\mu$ (GPa) & 0 & 30 \\
Viscous properties & 0 & 27 \\
$\quad$ Bulk viscosity $\eta_{\kappa}$ (GPa.day) & 0 & \\
Shear viscosity $\eta_{\mu}$ (GPa.day) & & \\
\hline
\end{tabular}

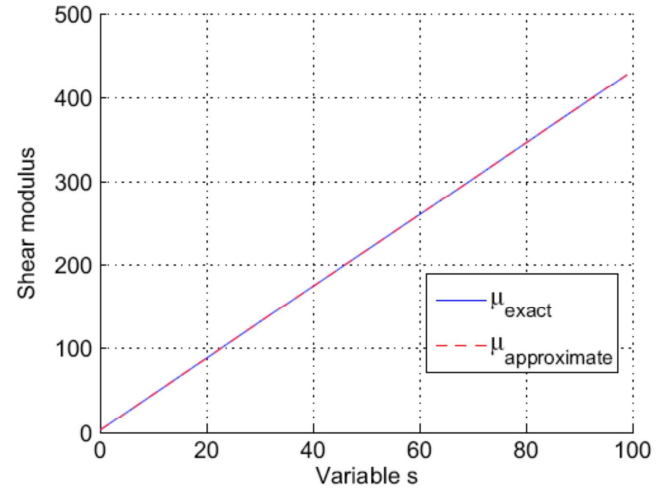

Fig. 13. Comparison between the exact solution and the RF approximate solution in Laplace-Carson's space for a Kelvin-Voigt viscoelastic matrix containing voided inclusions.

Fig. 17 provides again the comparison between the RF approximate values of the shear modulus in Laplace-Carson space with the exact value, showing again a good agreement. As for the case of voided inclusions, both results are located along a straight line, showing that the effective behavior is still of Kelvin-Voigt type, as for voided inclusions.

The relative error between exact and RF approximate solution in Laplace-Carson space is provided by Fig. 18, showing that the maximal error reaches now around $8 \cdot 10^{-4}$.

The values of the relaxation function obtained by the RF approximate solution and by the solution coming from the differential method are shown in Fig. 19. The results show again that the solution coming from the differential method over-evaluates the relaxation function.

Finally, Fig. 20 provides also the creep function obtained for the effective material, which is another quantity of interest.

\section{Evaluation of the accuracy of the RF approximate method}

The difference between the RF approximate solution and the exact solution of the Generalized self-consistent scheme depends of the properties of the constituents. This section intends to assess the error introduced by the RF approximate solution when using

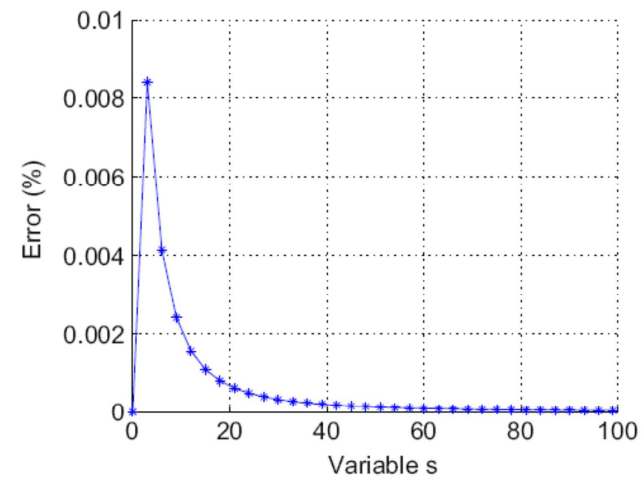

Fig. 14. Evaluation of the error coming from the RF approximate method in LaplaceCarson's space for a Kelvin-Voigt viscoelastic matrix containing voided inclusions. 


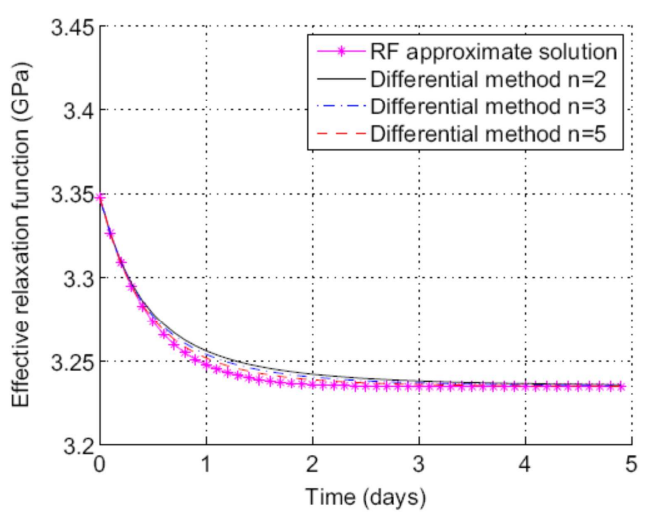

Fig. 15. Relaxation function in real space for a Kelvin-Voigt viscoelastic matrix containing voided inclusions.

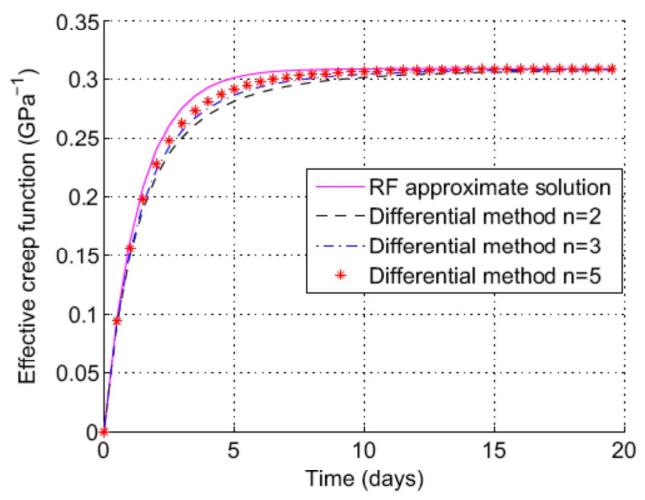

Fig. 16. Creep function in real space for a Kelvin-Voigt viscoelastic matrix containing voided inclusions.

different physical parameters. Given the number of parameters involved, the evaluation of this error will be performed assuming that the "Poisson's ratio" of the viscoelastic part is constant. Obviously, this assumption is not physically realistic. However, in numerous studied physical cases, it was observed that the error induced by the RF solution can be estimated by the one obtained for extremal values of the true Poisson's ratio over the physical range of this parameter. So, this approximation allows to evaluate the error coming from the approximate solution in the most general case.

In the most general case, the normalized effective modulus $\bar{\mu}_{\mathrm{eff}}$ depends on the transfer matrix [T] (Equation (B.48)). Given the assumption of "constant Poisson's ratio", this transfer matrix, for

Table 5

Volume fractions and material properties of the constituents of a 2-phase composite with rigid inclusions.

\begin{tabular}{llc}
\hline Material phases & Phase 1 & Phase 2 \\
\hline Volume fraction (\%) & 67 & 33 \\
Elastic properties & & 100 \\
Bulk modulus $\kappa$ (GPa) & $\infty$ & 20 \\
Shear modulus $\mu$ (GPa) & $\infty$ & 45 \\
Viscous properties & & 30 \\
$\quad$ Bulk viscosity $\eta_{\kappa}$ (GPa.day) & 0 & \\
Shear viscosity $\eta_{\mu}$ (GPa.day) & 0 & \\
\hline
\end{tabular}

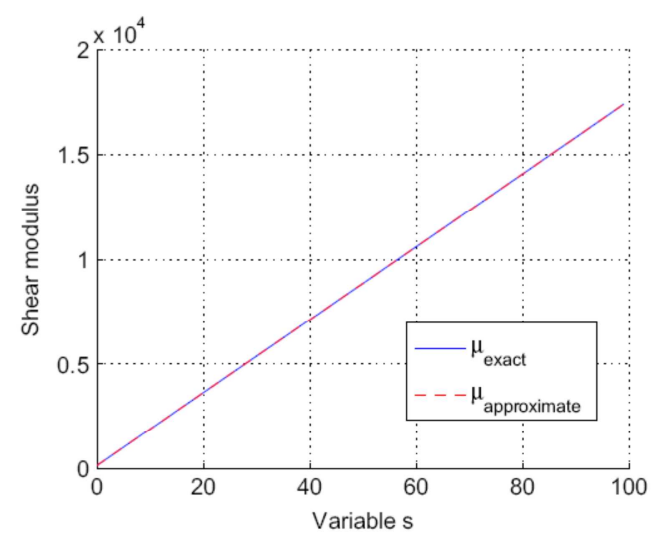

Fig. 17. Comparison between the exact solution and the RF approximate one in Laplace-Carson's space for a Kelvin-Voigt viscoelastic matrix containing rigid inclusions

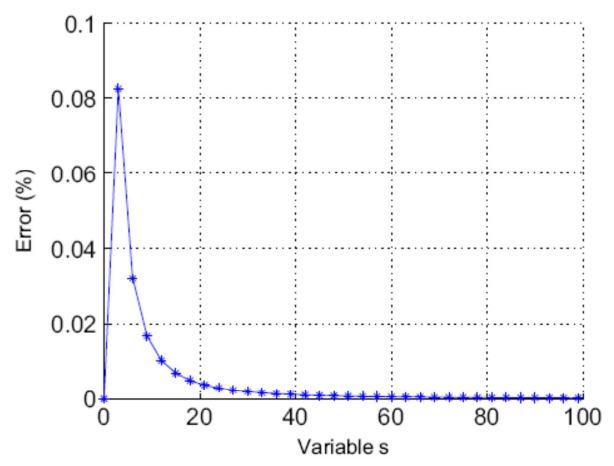

Fig. 18. Evaluation of the error coming from the RF approximate method in LaplaceCarson's space for a Kelvin-Voigt viscoelastic matrix containing rigid inclusions.

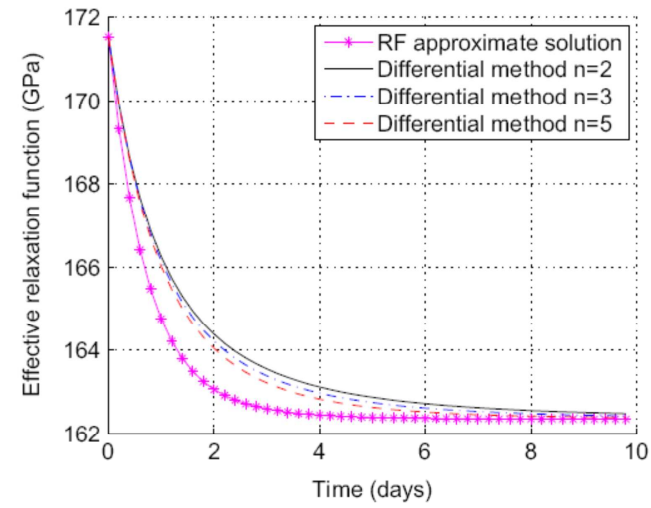

Fig. 19. Relaxation function in real space for a Kelvin-Voigt viscoelastic matrix containing rigid inclusions. 


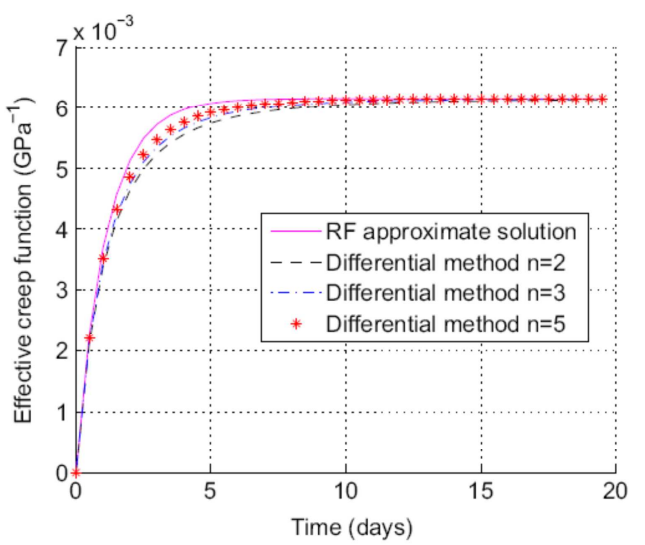

Fig 20. Creep function in real space for a Kelvin-Voigt viscoelastic matrix containing rigid inclusions.

the Kelvin-Voigt model, depends on five independent parameters: $f_{l}, \nu_{M}, v_{l}, \gamma, \xi$, whose last two ones are given by:

$\left\{\begin{array}{l}\gamma=\frac{\mu}{\mu_{1}} \\ \xi=\frac{\eta_{\mu}}{\mu_{1}}\end{array}\right.$

where $f_{L}$ is the concentration of inclusions, $\nu_{M}$ and $\nu_{I}$ are the Poisson's ratios of the phases, $\mu$ is the elastic part in the viscoelastic shear modulus of the matrix, $\mu_{1}$ is the shear modulus of the inclusion and $\eta_{\mu}$ is the viscosity in the rheological model related to shear.

In the following, three different values of Poisson's ratio are used: $0.1,0.3$, and 0.45 .

Fig. 21 displays, for a Kelvin-Voigt behavior of the matrix, the maximal errors (for values of $\gamma$ and $\xi$ between 0 and 1000) in Laplace's space coming from the RF approximate model as a function of the concentration and for various values of the Poisson's ratio of the phases. Similarly, Fig. 22 provides the results of the same systematic study for Maxwell type viscoelastic matrices.

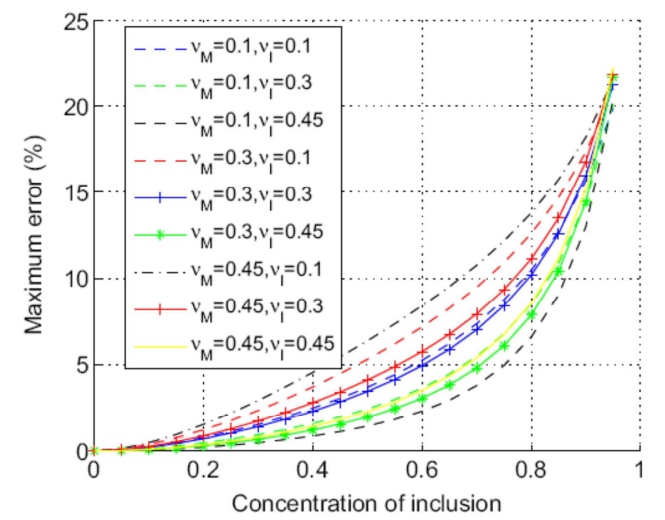

Fig 21. Evaluation of the maximal errors coming from the RF approximate method when using various physical parameters for Kelvin-Voigt viscoelastic materials.

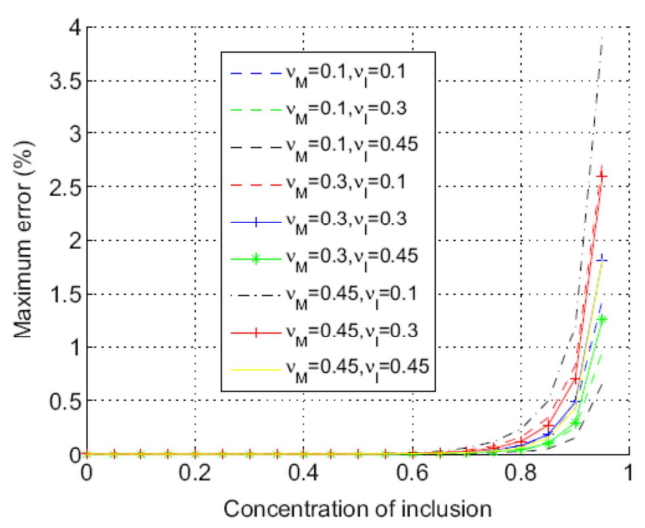

Fig. 22. Evaluation of the maximal errors coming from the RF approximate metho applied to various physical parameters for Maxwell type viscoelastic materials.

It is found that the error decreases with $\nu_{I}$ at $\nu_{M}$ constant and inversely increases with $\nu_{M}$ at $\nu_{I}$ constant. For Poisson's ratios being comparable in both phases and for Kelvin-Voigt's model, an error of less than 0.05 is provided for concentrations below 0.6. For the Maxwell's model, an error of less than $10^{-4}$ is provided for concentrations below 0.6 .

\section{Conclusion}

The Generalized self-consistent scheme is often used for predicting the behavior of matrix-inclusion composites. Using the correspondence principle allows the use of all computations performed for elastic materials for providing the values of effective parameters in Laplace-Carson space. The main difficulty is in providing the inverse Laplace transform. This paper has shown a simple way to provide an approximate solution by construction of a rational function which approximates the effective parameters in Laplace Carson's space. The method of construction allows to comply with both initial value and final value conditions. The results which were presented in the case of a viscoelastic matrix containing elastic inclusions have shown that this new approximate method provides satisfying solutions for Maxwell type and Kelvin-Voigt type materials.

\section{Appendix A. Inverse Laplace transform of a rational fractional function}

Solving the viscoelastic problem makes it necessary to inverse the Laplace transform from the Laplace space to real space. Let us consider the function in the Laplace space $F(s)$ which is a rational fraction having the numerator $P(s)$ and the denominator $Q(s)$ such that the degree of $P(s)$ is less than the degree of $Q(s)$. $Q(s)$ has $n$ distinct zeros $\alpha_{k}, k=1,2,3, \ldots, n$. Then:

$L^{-1}\{F(s)\}=L^{-1}\left\{\frac{P(s)}{Q(s)}\right\}=\sum_{k=1}^{n} \frac{P\left(\alpha_{k}\right)}{Q^{\prime}\left(\alpha_{k}\right)} e^{\alpha_{k} t}$

In the case where $Q(s)=0$ has a multiple root of order $p$, while the other roots, $\beta_{1}, \beta_{2}, \ldots, \beta_{n}$ are simple, the rational fraction becomes:

$\frac{P(s)}{Q(s)}=\sum_{k=1}^{p} \frac{A_{k}}{(s-\alpha)^{p-k+1}}+\sum_{j=1}^{n} \frac{B_{j}}{\left(s-\beta_{j}\right)}$ 
Then, the inverse of the Laplace transform becomes:

$L^{-1}\left\{\frac{P(s)}{Q(s)}\right\}=e^{\alpha t}\left\{\sum_{r=1}^{p} \frac{A_{r} t^{p-r}}{(p-r) !}\right\}+\sum_{r=1}^{n} B_{r} e^{\beta_{r} t}$

where:

$A_{k}=\lim _{s \rightarrow \alpha} \frac{1}{(k-1) !} \frac{\mathrm{d}^{k-1}}{\mathrm{~d} s^{k-1}}\left\{(s-\alpha)^{p} f(s)\right\}$

$B_{r}=\frac{P\left(\beta_{r}\right)}{Q^{\prime}\left(\beta_{r}\right)}$

Appendix B. Solutions of the Generalized self-consistent model for two-phase media

Appendix B.1. The bulk modulus

Let us consider the volume $\Omega$ defined in Fig. $1, R_{k}$ is the radius of the phase $k$. On the boundary $\partial \Omega$, the following displacement condition related to a homogeneous deformation is applied:

$\overline{\mathbf{u}}(x)=\varepsilon_{0} \mathbf{x}$

with $\varepsilon_{0}$ constant and symmetric.

The displacement and stress fields solutions in each phase $k$ are sought with the form:

$\left\{\begin{array}{l}u_{r}^{(k)}=A_{k} r+\frac{B_{k}}{r^{2}} \\ \sigma_{r r}^{(k)}=3 \kappa_{k} A_{k}-\frac{4 \mu_{k}}{r^{3}} B_{k} \\ \sigma_{\theta \theta}^{(k)}=3 \kappa_{k} A_{k}+\frac{2 \mu_{k}}{r^{3}} B_{k} \\ \sigma_{\phi \phi}^{(k)}=\sigma_{\theta \theta}^{(k)}\end{array}\right.$

The conditions of continuity of displacement and traction at the interphase between a phase $k$ and a phase $(k+1)$ can be written:

$\left\{\begin{array}{l}u_{r}^{(k+1)}\left(R_{k}\right)=u_{r}^{(k)}\left(R_{k}\right) \\ \sigma_{r+1)}^{(k+1)}\left(R_{k}\right)=\sigma_{r r}^{(k)}\left(R_{k}\right)\end{array}\right.$

which gives:

$\mathbf{J}_{k+1}\left(R_{k}\right)\left(\begin{array}{l}A_{k+1} \\ B_{k+1}\end{array}\right)=\mathbf{J}_{k}\left(R_{k}\right)\left(\begin{array}{l}A_{k} \\ B_{k}\end{array}\right)$

where the matrix $\mathbf{J}_{i}\left(R_{j}\right)$ is given by:

$\mathbf{J}_{i}\left(R_{j}\right)=\left[\begin{array}{cc}R_{j} & R_{j}^{-2} \\ 3 \kappa_{i} & -4 \mu_{i} R_{j}^{-3}\end{array}\right]$

For the first phase $(k=1)$ and for the effective medium $(k=n+1$ and $R_{n+1} \rightarrow \infty$ ), the value of strain field at the boundary and the condition for having a finite displacement at the center of the composite inclusion provide:

$\left\{B_{1}=0\right.$

$A_{n+1}=0$

In addition, writing that the elastic energy inside the composite inclusion is the same as the elastic energy within an inclusion containing a material having the properties of the effective medium (energetic neutrality) provides:

$B_{n+1}=0$

Relation B.4 can be considered as a recurrence relation. Combining the recurrence relations for all layers provides:

$\mathbf{J}_{n+1}\left(R_{n}\right)\left(\begin{array}{c}\varepsilon_{0} \\ 0\end{array}\right)=\mathbf{J}_{n}\left(R_{n}\right) \mathbf{Q}^{n-1}\left(\begin{array}{c}A_{1} \\ 0\end{array}\right)$

with:

$\left\{\begin{array}{l}\mathbf{Q}^{n-1}=\prod_{k=1}^{n-1} \mathbf{N}^{n-k+1} \\ \mathbf{N}^{k}=\mathbf{J}_{k+1}^{-1}\left(R_{k}\right) \mathbf{J}_{k}\left(R_{k}\right)\end{array}\right.$

Relation (B.8) leads to a system allowing the computation of $\kappa_{\mathrm{eff}}$ (which corresponds to $\kappa_{n}+1$ ):

$\left(\begin{array}{c}R_{n} \\ 3 \kappa_{\text {eff }}\end{array}\right) \varepsilon_{0}=\left[\begin{array}{cc}R_{n} & R_{n}^{-2} \\ 3 \kappa_{n} & -\frac{4 \mu_{n}}{R_{n}^{3}}\end{array}\right]\left(\begin{array}{c}Q_{11}^{n-1} \\ Q_{21}^{n-1}\end{array}\right) A_{1}$

which provides:

$\kappa_{\text {eff }}=\frac{3 \kappa_{n} R_{n}^{3} Q_{11}^{(n-1)}-4 \mu_{n} Q_{21}^{(n-1)}}{3\left(R_{n}^{3} Q_{11}^{(n-1)}+Q_{21}^{(n-1)}\right)}$

where the matrix involved in the calculation of the incompressibility modulus is given explicitly by:

$\mathbf{Q}^{(n-1)}=\prod_{k=1}^{n-1}\left[\begin{array}{ll}\frac{3 \kappa_{n-k}+4 \mu_{n-k+1}}{3 \kappa_{n-k+1}+4 \mu_{n-k+1}} & \frac{4\left(\mu_{n-k+1}-\mu_{n-k}\right) R_{n-k}^{-3}}{3 \kappa_{n-k+1}+4 \mu_{n-k+1}} \\ \frac{3\left(\kappa_{n-k+1}-\kappa_{n-k}\right) R_{n-k}^{3}}{3 \kappa_{n-k+1}+4 \mu_{n-k+1}} & \frac{3 \kappa_{n-k+1}+4 \mu_{n-k}}{3 \kappa_{n-k+1}+4 \mu_{n-k+1}}\end{array}\right]$

Appendix B.2. Shear modulus

Appendix B.2.1. General case

The displacement field is sought in every solid phase $k$ in the form:

$\left\{\begin{array}{l}u_{r}^{(k)}=U_{r}^{(k)}(r) \sin \theta^{2} \cos 2 \phi \\ u_{\theta}^{(k)}=U_{\theta}^{(k)}(r) \sin \theta \cos \theta \cos 2 \phi \\ u_{\phi}^{(k)}=U_{\phi}^{(k)}(r) \sin \theta \sin 2 \phi\end{array}\right.$

$\left\{\begin{array}{l}U_{r}^{(k)}(r)=A_{k} r-\frac{3 \kappa_{k}-2 \mu_{k}}{\mu_{k}} B_{k} r^{3}+\frac{3 C_{k}}{r^{4}}+3 \frac{\kappa_{k}+\mu_{k}}{\mu_{k}} \frac{D_{k}}{r^{2}} \\ U_{\theta}^{(k)}(r)=A_{k} r-\frac{15 \kappa_{k}+11 \mu_{k}}{3 \mu_{k}} B_{k} r^{3}-\frac{2 C_{k}}{r^{4}}+2 \frac{D_{k}}{r^{2}} \\ U_{\theta}^{(k)}(r)=-U_{\phi}^{(k)}(r)\end{array}\right.$

The integration constants $\left(A_{k}, B_{k}, C_{k}, D_{k}\right)$ are obtained as previously from the conditions at interfaces. For interfaces between 
phases $k$ and $k+1$ with $k=2 \ldots n$, we can write the classical continuity relations of traction and displacement:

$\mathbf{L}_{k+1}\left(R_{k}\right)\left(\begin{array}{c}A_{k+1} \\ B_{k+1} \\ C_{k+1} \\ D_{k+1}\end{array}\right)=\mathbf{L}_{k}\left(R_{k}\right)\left(\begin{array}{c}A_{k} \\ B_{k} \\ C_{k} \\ D_{k}\end{array}\right)$

where:

$\mathbf{L}_{k}(r)=\left[\begin{array}{llll}r & -\frac{\left(3 \kappa_{k}-2 \mu_{k}\right) r^{3}}{\mu_{k}} & \frac{3}{r^{4}} & 3 \frac{\left(\kappa_{k}+\mu_{k}\right)}{\mu_{k} r^{2}} \\ r & -\frac{\left(15 \kappa_{k}+11 \mu_{k}\right) r^{3}}{3 \mu_{k}} & \frac{-2}{r^{4}} & \frac{2}{r^{2}} \\ \mu_{k} & \frac{\left(3 \kappa_{k}-2 \mu_{k}\right) r^{2}}{2} & \frac{-12 \mu_{k}}{r^{5}} & -\frac{\left(9 \kappa_{k}+4 \mu_{k}\right)}{r^{3}} \\ \mu_{k} & -\frac{\left(24 \kappa_{k}+5 \mu_{k}\right) r^{2}}{3} & \frac{8 \mu_{k}}{r^{5}} & \frac{3 \kappa_{k}}{r^{3}}\end{array}\right]$

For the first phase $(k=1)$ and for the effective medium $(k=n+1)$, the condition for obtaining a finite value of displacement at the center and the condition of energetic neutrality provide:

$\left\{\begin{array}{l}A_{n+1}=\gamma_{0} \\ B_{n+1}=0 \\ C_{1}=0 \\ D_{1}=0, \quad D_{n+1}=0\end{array}\right.$

which gives:

$$
\begin{aligned}
& \left(\begin{array}{l}
\gamma_{0} \\
0 \\
C_{n+1} \\
0
\end{array}\right)=\mathbf{M}^{(n)} \ldots \mathbf{M}^{(k)} \ldots \mathbf{M}^{(1)}\left(\begin{array}{l}
A_{1} \\
B_{1} \\
0 \\
0
\end{array}\right) \\
& \left(\begin{array}{l}
\gamma_{0} \\
0 \\
C_{n+1} \\
0
\end{array}\right)=\mathbf{P}^{n}\left(\begin{array}{l}
A_{1} \\
B_{1} \\
0 \\
0
\end{array}\right)
\end{aligned}
$$

with:

$$
\left\{\begin{array}{l}
\mathbf{P}^{n}=\prod_{k=1}^{n} \mathbf{M}^{n-k+1} \\
\mathbf{M}^{k}=\mathbf{L}_{k+1}^{-1}\left(R_{k}\right) \mathbf{L}_{k}\left(R_{k}\right)
\end{array}\right.
$$

This homogeneous system of equations in $\gamma_{0}, C_{n+1}, A_{1}, B_{1}$ has a non-zero solution if its determinant is null, which provides a second order equation in $\mu_{\text {eff: }}$

$A\left(\frac{\mu_{\text {eff }}}{\mu_{n}}\right)^{2}+B\left(\frac{\mu_{\text {eff }}}{\mu_{n}}\right)+C=0$

where the coefficients are functions of elastic and geometric properties. However, in the values of $A, B, C$, all elastic coefficients are mixed. In order to replace the elastic coefficients by their viscoelastic counterparts in Laplace space, a different form of this system is written in the case of two phases, the new shape of relation allowing to express the coefficients as polynomials in the elastic properties.
Appendix B.2.2. Polynomial expression in elastic properties for the case of two phases $(n=2)$

As explained previously, the objective of the following is to split the contribution to the effective properties of the geometry and of the elastic properties of phases in the case of 2 phases. The continuity conditions of traction and displacement provide:

$\mathbf{L}_{\mathrm{eff}}\left(R_{2}\right)\left[\begin{array}{l}\gamma_{0} \\ 0 \\ C_{3} \\ 0\end{array}\right]=\mathbf{M}^{(2)} \mathbf{L}_{1}\left(R_{1}\right)\left[\begin{array}{l}A_{1} \\ B_{1} \\ 0 \\ 0\end{array}\right]$

This leads to:

$\left[\begin{array}{l}R_{2} \gamma_{0}+3 R_{2}^{-4} C_{3} \\ R_{2} \gamma_{0}-2 R_{2}^{-4} C_{3} \\ \mu_{\text {eff }}\left(\gamma_{0}-12 R_{2}^{-5} C_{3}\right) \\ \mu_{\text {eff }}\left(\gamma_{0}+8 R_{2}^{-5} C_{3}\right)\end{array}\right]=\mathbf{M}^{(2)} \mathbf{L}_{1}\left(R_{1}\right)\left[\begin{array}{l}A_{1} \\ B_{1} \\ 0 \\ 0\end{array}\right]$

Set $\mu=\mu_{2}, x=\kappa_{2} / \mu_{2}$, the matrix $\mathbf{L}_{2}(r)$ can be written:

$\mathbf{L}_{2}(r)=\mathbf{N}_{g}(r, \mu) \mathbf{L}_{2}^{*} \mathbf{N}_{d}(r)$

with:

$\mathbf{L}_{2}^{*}=\left[\begin{array}{llll}1 & 2-3 x & 3 & 3+3 x \\ 1 & -5 x-\frac{11}{3} & -2 & 2 \\ 1 & \frac{3 x}{2}-1 & -12 & -9 x-4 \\ 1 & -8 x-\frac{5}{3} & 8 & 3 x\end{array}\right]$

$\mathbf{N}_{g}(r, \mu)=\left[\begin{array}{cccc}1 & 0 & 0 & 0 \\ 0 & 1 & 0 & 0 \\ 0 & 0 & \frac{\mu}{r} & 0 \\ 0 & 0 & 0 & \frac{\mu}{r}\end{array}\right]$

$\mathbf{N}_{d}(r)=\left[\begin{array}{cccc}r & 0 & 0 & 0 \\ 0 & r^{3} & 0 & 0 \\ 0 & 0 & r^{-4} & 0 \\ 0 & 0 & 0 & r^{-2}\end{array}\right]$

then:

$\mathbf{L}_{2}\left(R_{2}\right) \mathbf{L}_{2}\left(R_{1}\right)^{-1}=\mathbf{N}_{g}\left(R_{2}, \mu\right) \mathbf{L}_{2}^{*} \times \mathbf{N}_{d}(\alpha)\left[\mathbf{L}_{2}^{*}\right]^{-1} \mathbf{N}_{g}\left(R_{1}, \mu\right)^{-1}$

with $\alpha=R_{2} / R_{1}$

The computation of the inverse of matrix $\mathbf{L}_{2}^{*}$, is given explicitly by:

$\left[\mathbf{L}_{2}^{*}\right]^{-1}=\frac{1}{5(3 x+4)} \times\left[\begin{array}{llll}18 x+8 & -9 x & 6 x+6 & 6 \\ \frac{24}{7} & -\frac{24}{7} & \frac{6}{7} & -\frac{6}{7} \\ \frac{3 x-2}{7} & \frac{-24 x-5}{7} & \frac{6 x-4}{7} & \frac{15 x+11}{7} \\ 2 & 3 & -2 & -3\end{array}\right]$ 
from which:

$\mathbf{L}_{2}^{*} \mathbf{N}_{d}(\alpha)\left(\mathbf{L}_{2}^{*}\right)^{-1}=\frac{\mathbf{C}_{k}(\alpha) x+\mathbf{C}_{\mu}(\alpha)}{5 \alpha^{4}(3 x+4)}$

(B.30)

where each matrix $\mathbf{C}_{\kappa}(\alpha)$ and $\mathbf{C}_{\mu}(\alpha)$ is given by polynomials in $\alpha$ with constant matrix coefficients:

$\mathbf{c}_{l}(\alpha)=\mathbf{c}_{10}+\mathbf{c}_{12} \alpha^{2}+\mathbf{c}_{15} \alpha^{5}+\mathbf{c}_{17} \alpha^{7}$

with $l=\kappa, \mu$

and:

$\mathbf{C}_{k 0}=\frac{3}{7}\left[\begin{array}{c}3 \\ -2 \\ -12 \\ 8\end{array}\right] \otimes\left[\begin{array}{c}1 \\ -8 \\ 2 \\ 5\end{array}\right]$

$\mathbf{C}_{k 2}=3\left[\begin{array}{c}1 \\ 0 \\ -3 \\ 1\end{array}\right] \otimes\left[\begin{array}{c}2 \\ 3 \\ -2 \\ -3\end{array}\right]$

$\mathbf{C}_{\kappa 5}=3\left[\begin{array}{l}1 \\ 1 \\ 1 \\ 1\end{array}\right] \otimes\left[\begin{array}{c}6 \\ -3 \\ 2 \\ 0\end{array}\right]$

$\mathbf{C}_{k 7}=\frac{3}{7}\left[\begin{array}{c}6 \\ 10 \\ -3 \\ 16\end{array}\right] \otimes\left[\begin{array}{c}-4 \\ 4 \\ -1 \\ 1\end{array}\right]$

$\mathbf{C}_{\mu 0}=\frac{1}{7}\left[\begin{array}{c}3 \\ -2 \\ -12 \\ 8\end{array}\right] \otimes\left[\begin{array}{l}-2 \\ -5 \\ -4 \\ 11\end{array}\right]$

$\mathbf{C}_{\mu 2}=\left[\begin{array}{c}3 \\ 2 \\ -4 \\ 0\end{array}\right] \otimes\left[\begin{array}{c}2 \\ 3 \\ -2 \\ -3\end{array}\right]$

$\mathbf{C}_{\mu 5}=2\left[\begin{array}{l}1 \\ 1 \\ 1 \\ 1\end{array}\right] \otimes\left[\begin{array}{l}4 \\ 0 \\ 3 \\ 3\end{array}\right]$

$\mathbf{C}_{\mu 7}=\frac{2}{7}\left[\begin{array}{c}-6 \\ 11 \\ 3 \\ 5\end{array}\right] \otimes\left[\begin{array}{c}-4 \\ 4 \\ -1 \\ 1\end{array}\right]$

We deduce:

$$
\begin{aligned}
{\left[\begin{array}{c}
\gamma_{0}+3 R_{2}^{-5} C_{3} \\
\gamma_{0}-2 R_{2}^{-5} C_{3} \\
\bar{\mu}_{\text {eff }}\left(\gamma_{0}-12 R_{2}^{-5} C_{3}\right) \\
\bar{\mu}_{\text {eff }}\left(\gamma_{0}+8 R_{2}^{-5} C_{3}\right)
\end{array}\right]=} & \frac{1}{5 \mu \alpha^{4}(3 \kappa+4 \mu)} \times\left(\kappa \mathbf{C}_{\kappa}(\alpha)+\mu \mathbf{C}_{\mu}(\alpha)\right) \\
& \times\left(\mu \mathbf{W}_{1}+R_{1} \mathbf{W}_{0}\right) \mathbf{L}_{1}\left(R_{1}\right)\left[\begin{array}{c}
A_{1} \\
B_{1} \\
0 \\
0
\end{array}\right]
\end{aligned}
$$

with:

$\bar{\mu}_{\text {eff }}=\frac{\mu_{\text {eff }}}{\mu_{2}}$

$\mathbf{W}_{1}=\left[\begin{array}{llll}1 & 0 & 0 & 0 \\ 0 & 1 & 0 & 0 \\ 0 & 0 & 0 & 0 \\ 0 & 0 & 0 & 0\end{array}\right]$

$\mathbf{W}_{0}=\left[\begin{array}{llll}0 & 0 & 0 & 0 \\ 0 & 0 & 0 & 0 \\ 0 & 0 & 1 & 0 \\ 0 & 0 & 0 & 1\end{array}\right]$ Set $C_{3}^{\prime}=R_{2}^{-5} C_{3}$ and $A_{1}^{\prime}=A_{1} / 5 \mu \alpha^{4} R_{2}(3 \kappa+4 \mu)$ :

$\left[\begin{array}{c}\gamma_{0}+3 C_{3}^{\prime} \\ \gamma_{0}-2 C_{3}^{\prime} \\ \bar{\mu}_{\mathrm{eff}}\left(\gamma_{0}-12 C_{3}^{\prime}\right) \\ \bar{\mu}_{\mathrm{eff}}\left(\gamma_{0}+8 C_{3}^{\prime}\right)\end{array}\right]=\left(\kappa \mathbf{C}_{\kappa}(\alpha)+\mu \mathbf{C}_{\mu}(\alpha)\right)$

$$
\times\left(\mu \mathbf{W}_{1}+R_{1} \mathbf{W}_{0}\right) \mathbf{L}_{1}\left(R_{1}\right)\left[\begin{array}{c}
A_{1}^{\prime} \\
B_{1}^{\prime} \\
0 \\
0
\end{array}\right]
$$

$\mathbf{L}_{1}\left(R_{1}\right)=\mathbf{N}_{g}\left(\mu_{1}, R_{1}\right) \mathbf{L}_{1}^{*}\left(x_{1}\right) \mathbf{N}_{d}\left(R_{1}\right)$

The matrix $\mathbf{N}_{d}\left(R_{1}\right)$ is diagonal which provides:

$\mathbf{N}_{d}\left(R_{1}\right)\left[\begin{array}{c}A_{1}^{\prime} \\ B_{1}^{\prime} \\ 0 \\ 0\end{array}\right]=\left[\begin{array}{c}A_{1}^{\prime \prime} \\ B_{1}^{\prime \prime} \\ 0 \\ 0\end{array}\right]$

with: $A_{1}^{\prime \prime}=R_{1} A_{1}^{\prime}$ et $B_{1}^{\prime \prime}=R_{1}^{3} B_{1}^{\prime \prime}$

As a result:

$\left[\begin{array}{c}\gamma_{0}+3 C_{3}^{\prime} \\ \gamma_{0}-2 C_{3}^{\prime} \\ \bar{\mu}_{\mathrm{eff}}\left(\gamma_{0}-12 C_{3}^{\prime}\right) \\ \bar{\mu}_{\mathrm{eff}}\left(\gamma_{0}+8 C_{3}^{\prime}\right)\end{array}\right]=\mathbf{T}\left[\begin{array}{c}A_{1}^{\prime \prime} \\ B_{1}^{\prime \prime} \\ 0 \\ 0\end{array}\right]$

where

$\mathbf{T}=\left(\kappa \mathbf{C}_{\kappa}(\alpha)+\mu \mathbf{C}_{\mu}(\alpha)\right)\left(\mu \mathbf{W}_{1}+\mu_{1} \mathbf{W}_{0}\right) \mathbf{L}_{1}^{*}\left(x_{1}\right)$

The determinant of this system for unknowns $\gamma_{0}, C_{n+1}^{\prime}, A_{1}^{\prime \prime}, B_{1}^{\prime \prime}$ must be null for having a non-zero solution, which leads to a second order equation where the overall modulus normalized shear $\bar{\mu}_{\mathrm{eff}}$ is obtained by:

$A\left(\bar{\mu}_{\text {eff }}\right)^{2}+B \bar{\mu}_{\mathrm{eff}}+C=0$

(B.49)

with:

$\left\{\begin{array}{l}A=4 \Delta_{12} \\ B=\Delta_{23}-2 \Delta_{13}+3 \Delta_{24}-2 \Delta_{14} \\ C=-\Delta_{34}\end{array}\right.$ 
where $\Delta_{k l}$ is determined from the coefficients of the transfer matrix by:

$\Delta_{k l}=\left|\begin{array}{cc}T_{k 1} & T_{k 2} \\ T_{l 1} & T_{l 2}\end{array}\right|$

For a material where the matrix is viscoelastic of Kelvin-Voigt type, the pseudo-elastic coefficients in Laplace-Carson space are:

$\left\{\begin{array}{l}R_{\kappa}^{*(2)}(s)=K\left(1+\tau_{\kappa} S\right) \\ R_{\mu}^{*(2)}(s)=G\left(1+\tau_{\mu} s\right)\end{array}\right.$

where $K$ and $G$ are the elastic moduli of spring, $\tau_{K}$ and $\tau_{\mu}$ are the relaxation times of the dashpot in compression and shear respectively.

In this case, we find that the matrix $\mathbf{T}^{*}(s)$ has degree 2 , then $\Delta_{k l}$ has degree 4 in $s$. Then we deduce that $A^{*}(s), B^{*}(s), C^{*}(s)$ are polynomials of degree 4 in $s$.

Appendix B.2.3. Voided inclusion $(n=2)$

In the case of voided inclusion $\left(\mu_{1}=0\right)$, the transfer matrix $\mathbf{T}$ becomes:

$\mathbf{T}=\left(\kappa \mathbf{C}_{k}(\alpha)+\mu \mathbf{C}_{\mu}(\alpha)\right) \mu \mathbf{W}_{1} \mathbf{L}_{1}^{*}\left(x_{1}\right)$

Then the latter product is equal to:

$\mu \mathbf{W}_{1} \mathbf{L}_{1}^{*}\left(x_{1}\right)\left[\begin{array}{c}A_{1}^{\prime \prime} \\ B_{1}^{\prime \prime} \\ 0 \\ 0\end{array}\right]=\left[\begin{array}{c}C \\ D \\ 0 \\ 0\end{array}\right]$

The treatment is identical to the previous case with the transfer matrix :

$\mathbf{T}_{0}=\kappa \mathbf{C}_{\kappa}(\alpha)+\mu \mathbf{C}_{\mu}(\alpha)$

For the Kelvin-Voigt viscoelastic material, $\mathbf{T}_{0}^{*}(s)$ has degree 1 . Then $A^{*}(s), B^{*}(s), C^{*}(s)$ are polynomials of degree 2 in $s$.

Appendix B.2.4. Rigid inclusion $(n=2)$

For this case, $\mu_{1} \gg \mu$ the transfer matrix becomes:

$\mathbf{T}_{\infty}=\left(\kappa \mathbf{C}_{\kappa}(\alpha)+\mu \mathbf{C}_{\mu}(\alpha)\right) \mu_{1} \mathbf{W}_{0} \mathbf{L}_{1}^{*}\left(x_{1}\right)$

The final product becomes:

$\mu_{1} \mathbf{W}_{0} \mathbf{L}_{1}^{*}\left(x_{1}\right)\left[\begin{array}{c}A_{1}^{\prime \prime} \\ B_{1}^{\prime \prime} \\ 0 \\ 0\end{array}\right]=\left[\begin{array}{c}0 \\ 0 \\ E \\ F\end{array}\right]$

An alternative is to keep the same procedure as before with the construction from $\Delta_{k}$ in (B.51) by adopting the matrix:

$\mathbf{T}_{\infty}=\left(\kappa \mathbf{C}_{\kappa}(\alpha)+\mu \mathbf{C}_{\mu}(\alpha)\right) \mathbf{P}$

where the matrix $\mathbf{P}$ is the permutation matrix:

$\mathbf{P}=\left[\begin{array}{llll}0 & 0 & 1 & 0 \\ 0 & 0 & 0 & 1 \\ 1 & 0 & 0 & 0 \\ 0 & 1 & 0 & 0\end{array}\right]$

For the Kelvin-Voigt viscoelastic material, $\mathbf{T}_{0}^{*}(s)$ has degree 1 . Then $A^{*}(s), B^{*}(s), C^{*}(s)$ have degree 2 in $s$.
Appendix C. Decomposition of elasticity and viscoelasticity

tensors for a Kelvin-Voigt material

Appendix C.1. Elastic behavior

Let us consider a material having an isotropic elastic behavior, defined by the shear moduli $\mu$ and by the incompressibility $\kappa$. Then, the stiffness tensor $\mathbb{C}$ and the compliance tensor $\mathbb{S}$ are determined (see for example (Zaoui and Ecole polytechnique, 1998)) by:

$\left\{\begin{array}{l}\mathbb{C}=2 \mu \mathbb{K}+3 \kappa \mathbb{J} \\ \mathbb{S}=\frac{1}{2 \mu} \mathbb{K}+\frac{1}{3 \kappa} \mathbb{J}\end{array}\right.$

where the fourth-order tensors $\mathbb{K}$ and $\rrbracket$ are spherical and deviatoric parts, respectively, and defined by:

$\left\{\begin{array}{l}J=\frac{1}{3} \mathbf{i} \otimes \mathbf{i} \\ \mathbb{N}=\mathbb{\mathbb { V }}-\mathbb{J}\end{array}\right.$

i and $\mathbb{n}$ being the second-order and fourth-order identity tensors The fourth-order tensors $\mathbb{K}$ and $\mathbb{}$ have the following properties:

$\left\{\begin{array}{lll}\mathbb{U}: \mathbb{}=\mathbb{V} & \mathbb{K}: \mathbb{K}=\mathbb{K} & \mathbb{J}: \mathbb{}=\mathbb{J} \\ \mathbb{K}: \mathbb{J}=0 & \mathbb{0}: \mathbf{i}=\mathbb{J}: \mathbf{i}=\mathbf{i} & \mathbb{K}: \mathbf{i}=0 \\ \mathbb{J}: \mathbb{K}=0 & \mathbb{K}: \varepsilon=\mathbf{e} & \mathbb{\varepsilon}: \varepsilon=\frac{1}{3} \operatorname{tr}(\varepsilon) \mathbf{i}\end{array}\right.$

where the tensor $\mathbf{e}$ is the deviator of a second order symmetric tensor $\varepsilon$.

Appendix C.2. Viscoelastic constitutive equations for the KelvinVoigt's viscoelastic material by:

The viscoelastic Kelvin-Voigt constitutive equations are given

$\boldsymbol{\sigma}=\mathbb{C}: \varepsilon+\boldsymbol{\eta}: \dot{\varepsilon}$

where $\eta$ is the fourth-order tensor, defined by:

$\eta=3 \eta_{\kappa} \mathbb{J}+2 \eta_{\mu} \mathbb{K}$

In the Laplace-Carson's space, this can be written:

$\boldsymbol{\sigma}^{*}=(\mathbb{C}+\boldsymbol{\eta} s): \varepsilon^{*}$

which gives:

$\boldsymbol{\sigma}^{*}=\left[3\left(\kappa+\eta_{\kappa} s\right) \mathbb{J}+2\left(\mu+\eta_{\mu} s\right) \mathbb{K}\right]: \varepsilon^{*}$

or:

$\boldsymbol{\sigma}^{*}=\mathbb{R}^{*}: \varepsilon^{*}$

with:

$\mathbb{R}^{*}=3 R_{\kappa}^{*} \unlhd+2 R_{\mu}^{*} \mathbb{K}$

and:

$\left\{R_{\kappa}^{*}=\kappa+\eta_{\kappa} s\right.$

$\left\{R_{\mu}^{*}=\mu+\eta_{\mu} S\right.$

where $\eta_{\kappa}, \eta_{\mu}$ are the shear and compression viscosities. 
Appendix C.3. Viscoelastic behavior of the Maxwell's model

The viscoelastic behavior of Maxwell type is given by:

$\dot{\varepsilon}=\mathbb{C}^{-1}: \dot{\boldsymbol{\sigma}}+\eta^{-1}: \boldsymbol{\sigma}$

This provides in Laplace-Carson's space:

$\varepsilon^{*}=\left(\mathbb{C}^{-1}+\frac{1}{s} \eta^{-1}\right): \sigma^{*}$

which gives:

$\varepsilon^{*}=\left[\left(\frac{1}{3 \kappa}+\frac{1}{3 \eta_{\kappa} s}\right) \unlhd+\left(\frac{1}{2 \mu}+\frac{1}{2 \eta_{\mu} s}\right) \mathbb{K}\right]: \boldsymbol{\sigma}^{*}$

or:

$\boldsymbol{\sigma}^{*}=\mathbb{R}^{*}: \varepsilon^{*}$

where:

$\left\{\begin{array}{l}R_{\kappa}^{*}=\frac{\kappa \eta_{\kappa} s}{\kappa+\eta_{\kappa} s} \\ R_{\mu}^{*}=\frac{\mu \eta_{\mu} s}{\mu+\eta_{\mu} s}\end{array}\right.$

\section{References}

Beurthey, S., Zaoui, A., 2000. Structural morphology and relaxation spectra of viscoelastic heterogeneous materials. European Journal of Mechanics - A/Solids $19(1), 1-16$

Brinson, L.C., Lin, W.S., 1998. Comparison of micromechanics methods for effective properties of multiphase viscoelastic composites. Composite Structures $41(3-4), 353-367$
Christensen, R.M., Freund, L.B., 1984. Theory of viscoelasticity, an introduction. Journal of Applied Mechanics 51, 226

Christensen, R.M., Lo, K.H., 1979. Solutions for effective shear properties in three phase sphere and cylinder models. Journal of the Mechanics and Physics of Solids 27 (4), 315-330.

Donolato, C., 2002. Analytical and numerical inversion of the Laplace-Carson transform by a differential method. Computer Physics Communications 145 (2), 298-309.

Eshelby, J.D., 1956. The continuum theory of lattice defects. Solid State Physics 3 , 79-144.

Herve, E., Zaoui, A., 1993. N-layered inclusion-based micromechanical modelling. International Journal of Engineering Science $31(1), 1-10$.

Le, Q.V., Meftah, F., He, Q.C., Le Pape, Y., 2007. Creep and relaxation functions of a heterogeneous viscoelastic porous medium using the Mori-Tanaka homogenization scheme and a discrete microscopic retardation spectrum. Mechanics of Time-dependent Materials 11 (3), 309-331.

Lee, E.H., 1955. Stress analysis in visco-elastic bodies. Quarterly of Applied Mathematics $13,183-190$

Mandel, J., 1966. Cours de mécanique des milieux continus. Gauthier-Villars.

Masson, R., Brenner, R., Castelnau, O., 2012. Incremental homogenization approach for ageing viscoelastic polycrystals. Comptes Rendus Mćanique 340 (4-5), 378386.

Mori, T., Tanaka, K., 1973. Average stress in matrix and average elastic energy of materials with misfitting inclusions. Acta Metallurgica 21 (5), 571-574.

Nguyen, S.T., Dormieux, L., Le Pape, Y., Sanahuja, J., 2010. Crack propagation in viscoelastic structures: theoretical and numerical analyses. Computational Materials Science 50 (1), 83-91.

Rekik, A., Brenner, R., 2011. Optimization of the collocation inversion method for the linear viscoelastic homogenization. Mechanics Research Communications 38 (4), 305-308.

Rougier, Y., Stolz, C., Zaoui, A., 1994. Self-consistent modelling of elastic-viscoplastic polycrystals. Comptes rendus de l'Académie des sciences. Série 2, Mécanique, Physique, Chimie, Sciences de l'univers, Sciences de la Terre 318 (2), 145-151. Salençon, J., 2009. Viscoélasticité pour le calcul des structures. Editions Ecole Polytechnique

Schapery, R.A., 1962. Approximate methods of transform inversion for viscoelastic stress analysis. In: Proc. U.S. Nat. Congr. Appl. Mech. ASME 4th, vol. 2, pp. 10751085

Selivanov, M.F., Chernoivan, Y.A., 2007. A combined approach of the Laplace transform and Padé approximation solving viscoelasticity problems. International Journal of Solids and Structures 44 (1), 66-76.

Wang, Y.M., Weng, G.J., 1992. The influence of inclusion shape on the overall viscoelastic behavior of composites. Journal of Applied Mechanics 59 (3), $510-$ 518

aoui, A., Ecole polytechnique (France), 1998. Département de physique. Matériaux hétérogènes et composites. Ecole polytechnque. 